\title{
Emergency pediatric radiology imaging trends for non-COVID-19-related illnesses through different stages of the pandemic
}

\author{
Wendy G. Kim ${ }^{1}$ D . Stephen D. Brown ${ }^{1} \cdot$ Patrick R. Johnston ${ }^{1} \cdot$ Joshua Nagler $^{2} \cdot$ Delma Y. Jarrett $^{3}$
}

Received: 16 August 2021 / Accepted: 20 October 2021 / Published online: 2 November 2021

(c) American Society of Emergency Radiology 2021

\begin{abstract}
Purpose To evaluate how the COVID-19 pandemic affected the imaging utilization patterns for non-COVID-19-related illness in a pediatric emergency department (ED).

Methods We retrospectively reviewed radiology reports for ultrasound, CT, MRI, and fluoroscopy studies performed at a pediatric ED in April from 2017 to 2021, excluding studies for respiratory symptoms and trauma. Radiology reports and medical records were reviewed to determine if patients had a positive radiology diagnosis, the type of diagnosis, and whether it required hospital admission. Results from during the pandemic were compared to predicted rates based on pre-pandemic years.

Results A total of 2198 imaging studies were included. During the COVID-19 pandemic, fewer ED imaging studies were performed compared to predicted. The decrease was greater in April $2020(\mathrm{RR}=0.56, p<0.001)$ than in April $2021(\mathrm{RR}=0.80$, $p=0.038$ ). The odds of positive diagnosis was higher during the pandemic than before, and higher in 2020 (OR 2.53, $p<0.001$ ) than in 2021 (OR 1.38, $p=0.008$ ). The expected numbers of positive diagnoses and hospital admittances remained within the predicted range during the pandemic $(p=0.505-0.873)$.

Conclusions Although imaging volumes decreased during the studied months of the pandemic, the number of positive findings was unchanged compared to prior years. No differences were demonstrated in the percentage of patients admitted to the hospital with positive imaging findings. This suggests that, at our institution, the pandemic did not lead to a substantial number of missed diagnoses or severely delay the diagnosis of non-COVID-related conditions. While still lower than expected, imaging volumes increased in April 2021 suggesting a return towards baseline patient behavior as the pandemic conditions improved.
\end{abstract}

Keywords COVID-19 · Pediatric · Emergency Department, Utilization

\section{Introduction}

Wendy G. Kim

wendy.kim@childrens.harvard.edu

1 Department of Radiology, Boston Children's Hospital, Harvard Medical School, 300 Longwood Avenue, Boston, MA 02115, USA

2 Division of Emergency Medicine, Boston Children's Hospital, Harvard Medical School, Boston, MA, USA

3 Department of Radiology, Weill Cornell Medicine, New York-Presbyterian Hospital, New York, NY, USA
The novel coronavirus disease 2019 (COVID-19) was declared a pandemic on March 11, 2020, by the World Health Organization. Soon after, the United States (US) declared a national emergency. To curb pandemic spread, many states enforced mandatory stay-at-home orders in March 2020, which limited gatherings and temporarily closed non-essential businesses and organizations [1]. Additionally, the Centers for Disease Control (CDC) recommended that healthcare systems prioritize urgent visits and delay elective care [2]. Many healthcare facilities were urged to reallocate resources in preparation for the impending rise in hospital admissions related to COVID-19, and also to encourage patients to use telehealth services instead of seeking in-person medical care 
for preventative and non-emergent care [3]. Subsequently, the volume of patients seeking emergency medical care in the US decreased dramatically during the early stages of the pandemic, with the largest decreases observed in April 2020, in the most severely impacted areas [4].

Several adult and pediatric studies in the US and similarly affected European countries have shown that the decrease in patient volume was associated with an unexpected decrease in the number of patients presenting to the emergency department (ED) with non-COVID-associated emergencies including acute coronary syndromes, strokes, and appendicitis [5-8]. Of the adult patients who were diagnosed with non-COVID emergencies, many exhibited more acute presentations and increased complications, such as gastrointestinal perforations, septicemia, and increased hospital mortality after cardiovascular emergencies [9-11]. These worrisome trends suggest that many adult patients with serious medical conditions either delayed medical care or did not seek appropriate medical attention at all. A small number of studies raised concerns for similar trends in the pediatric population $[12,13]$. The objective of this study is to further explore this question by evaluating the effect of the COVID-19 pandemic on pediatric emergency imaging for non-COVID-related indications. In it, we evaluate the number of imaging studies performed, as well as the types and severity of radiologic findings during two different phases of the COVID-19 pandemic: a 1-month period in the first wave of the pandemic, with stay-at-home orders in place, and a 1-month period, 1 year later, during the 3rd wave of the pandemic, after stay-at-home orders had been lifted. We compare these months to the same month in years preceding the pandemic.

\section{Methods}

The study was performed at a single, urban, academic children's hospital with over 400 beds. The institutional review board granted an exemption, and written patient or guardian consent was waived. We retrospectively reviewed all radiology reports for advanced imaging studies (ultrasound, computed tomography (CT), magnetic resonance (MR), and fluoroscopy) performed on emergency department patients of all ages during the month of April, for the years 2017 through 2021. April 2020 was the first full month of the COVID-19 pandemic, with the most dramatic decrease in the volume of patients seeking emergency medical care [4, 5, 14]. Similarly, at our institution, April 2020 showed the lowest ED patient volume between January 2017 and June 2021, with only 2185 total patients compared to an average volume of about 5000 patients per month over the 3 years preceding the pandemic. We included all internally generated reports for studies performed at our institution and those performed at outside institutions for which a second opinion consultation report was requested by the ED staff. These studies were identified by searching radiology reports for the included dates and modalities using Nuance $\AA$ PowerScribe ${ }^{\circledR} 360$ Reporting and Montage ${ }^{\circledR}$ Search and Analytics tools.

The clinical indications for the imaging studies were reviewed. We excluded imaging studies requested for evaluation of airway or respiratory symptoms, because these might coincide with studies evaluating patients for COVID-19. We also excluded imaging studies requested for trauma evaluation to avoid the confounding effect of decreased trauma rates associated with stay-at-home orders [15-17]. Radiographs were excluded because most chest and extremity radiographs in our ED are performed to evaluate for respiratory symptoms or trauma.

We reviewed individual radiology reports and designated each study as either a positive diagnosis, based on abnormal imaging findings that would account for the patient's presenting symptoms, or a negative diagnosis. If a patient underwent multiple imaging studies to evaluate for the same diagnosis, only one study was counted, with priority given to a positive exam, and the other exams were excluded. However, if a patient had two imaging studies for different indications (involving different anatomic locations), both studies were included. Positive studies were further categorized as those that required hospital admission and those that did not. All positive studies were categorized by diagnosis type (Table 1). Acute appendicitis (AA) was subcategorized separately because it is a relatively common, time-sensitive diagnosis where delayed presentation and treatment could result in increased complications or worse outcomes [18].

\section{Statistical analysis}

The study's main outcome variables were imaging frequency, diagnosis, and admission, all potentially depending on year. Frequency data was fit by Poisson exponential models to estimate rates (mean frequency per year), and binary data was fit by binomial logistic models to estimate odds and probabilities of events [19]. Comparisons were via ratios of rates (RR) for Poisson models and via ratios of odds (OR) for binomial models. Statistical significance for testing the equality of rates and odds was at the 5\% level.

Regression-type models (treating year numerically as 0-4 for years 2017-2021) were used to predict 2020 and 2021 rates and odds based on 2017-2019 data using SAS's NLMIXED (nonlinear mixed models) procedure [20]. These predictions were compared to actual observed data for 2020 or 2021 based on a comparison of two independent rates or odds. Regression models for all outcomes except diagnosis odds included a predictor for year. For diagnosis odds, the Akaike information criterion (AIC) favored the simpler 
Table 1 Diagnosis categories and rates

\begin{tabular}{|c|c|c|c|c|c|c|}
\hline \multirow[b]{2}{*}{ Category } & \multirow[b]{2}{*}{ Types of diagnoses included } & \multicolumn{5}{|c|}{ Number of cases per year } \\
\hline & & 2021 & 2020 & 2019 & 2018 & 2017 \\
\hline \multirow[t]{2}{*}{ Gastrointestinal illnesses (GI) } & $\begin{array}{l}\text { Intussusception, bowel obstruction, inflammatory or infectious bowel dis- } \\
\text { ease, malrotation, esophageal foreign body, acute cholecystitis, pancreatitis, } \\
\text { intra-abdominal abscess }\end{array}$ & 43 & 51 & 39 & 32 & 19 \\
\hline & Acute appendicitis (AA) & 22 & 33 & 26 & 18 & 12 \\
\hline Soft tissue abnormalities (ST) & Osteomyelitis, myositis, synovitis, joint effusion, cellulitis, abscess & 15 & 18 & 11 & 13 & 11 \\
\hline Head and neck infections (HN) & $\begin{array}{l}\text { Cervical lymphadenitis, sialoadenitis, retropharyngeal and parapharyngeal } \\
\text { infections, orbital infections, acute sinusitis, mastoiditis, otitis, thyroiditis, } \\
\text { odontogenic infections }\end{array}$ & 13 & 11 & 19 & 14 & 21 \\
\hline Neurologic abnormalities (N) & $\begin{array}{l}\text { Ventriculomegaly, intracranial hemorrhage, stroke, cerebritis, cerebral } \\
\text { edema, optic neuritis, spinal cord infections/inflammation }\end{array}$ & 31 & 7 & 11 & 8 & 14 \\
\hline Oncologic diagnoses $(\mathrm{ON})$ & New diagnosis and complications of existing diagnosis & 6 & 7 & 3 & 6 & 5 \\
\hline Hematologic abnormalities $(\mathrm{H})$ & $\begin{array}{l}\text { Deep venous thrombosis, pulmonary embolism, vascular and lymphatic } \\
\text { malformations, other bleeding disorder }\end{array}$ & 2 & 3 & 5 & 0 & 0 \\
\hline Genitourinary diagnoses (GU) & $\begin{array}{l}\text { Epididymitis, orchitis, torsed appendage, renal stones, pyelonephritis, salpin- } \\
\text { gitis, tubo-ovarian abscess, ectopic pregnancy, gonadal torsion }\end{array}$ & 24 & 30 & 16 & 14 & 15 \\
\hline Total & & 134 & 127 & 104 & 87 & 85 \\
\hline
\end{tabular}

model $(\mathrm{AIC}=21)$ to the model including year $(\mathrm{AIC}=23)$ [21].

\section{Results}

A total of 2198 imaging studies were included in the analysis. The distribution of frequencies over diagnosis categories is listed in Table 1. Gastrointestinal disease was the most common diagnosis category in aggregate over 2017-2021 and appendicitis was the most common individual diagnosis for each of the 5 years. Rates and odds for 2017-2021 are summarized in Table 2.

We predicted increased imaging rates for 2020 and 2021 based on the observed trend of rising imaging rates from 2017 to 2019 . Imaging rates in 2020 and 2021 were both lower than predicted, with a more pronounced decrease in $2020(\mathrm{RR}=324 / 574=0.56, p<0.001)$ than in 2021 $(\mathrm{RR}=515 / 647=0.80, p=0.038)$ (Fig. 1$)$.

Compared to predictions based on increasing trends over 2017-2019, the number of patients with a positive diagnosis on an ED imaging exam did not significantly change in either $2020(\mathrm{RR}=1.13, p=0.505)$ or 2021 ( $\mathrm{RR}=1.07$, $p=0.774$ ) (Fig. 2A). Because imaging rates were lower than predicted, the odds of a positive diagnosis were significantly higher than predicted in both $2020(\mathrm{OR}=0.64 / 0.25=2.53$, $p<0.001)$ and $2021(\mathrm{OR}=0.35 / 0.25=1.38, p=0.008)$ (Fig. 2B).

Compared to predictions based on 2017-2019 data, the change in admission rates was not significant in either 2020 $(\mathrm{RR} 83 / 80=1.04, p=0.873)$ or $2021(\mathrm{RR}=85 / 97=0.88$, $p=0.668$ ) (Fig. 3A). The change in the odds of admission
Table 2 Rates and odds for 2017-2021

\begin{tabular}{llllll}
\hline Year & Imaging rate & Diagnosis rate & Admission rate & Diagnosis odds & Admission odds \\
\hline 2017 & 395 & 85 & 46 & 0.27 & 1.18 \\
2018 & 460 & 87 & 53 & 0.23 & 1.56 \\
2019 & 504 & 104 & 67 & 0.26 & 1.81 \\
2020 & 324 & 127 & 83 & 0.64 & 1.89 \\
2021 & 515 & 134 & 85 & 0.35 & 1.73 \\
Pred $(2020)^{\mathrm{a}}$ & 574 & 113 & 80 & 0.25 & 2.28 \\
Pred (2021) & 647 & 125 & 97 & 0.25 & 2.83 \\
2020 vs Pred & $\mathrm{RR}=0.56$ & $\mathrm{RR}=1.13$ & $\mathrm{RR}=1.04$ & $\mathrm{OR}=2.53$ & $\mathrm{OR}=0.83$ \\
& $p<0.001$ & $p=0.505$ & $p=0.873$ & $p<0.001$ & $p=0.604$ \\
2021 vs Pred & $\mathrm{RR}=0.80$ & $\mathrm{RR}=1.07$ & $\mathrm{RR}=0.88$ & $\mathrm{OR}=1.38$ & $\mathrm{OR}=0.61$ \\
& $p=0.038$ & $p=0.774$ & $p=0.668$ & $p=0.008$ & $p=0.322$ \\
\hline
\end{tabular}

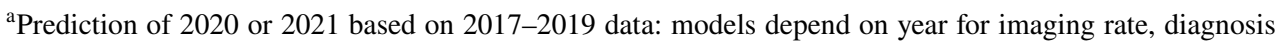
rate, admission rate, and admission odds, and do not depend on year for diagnosis odds 


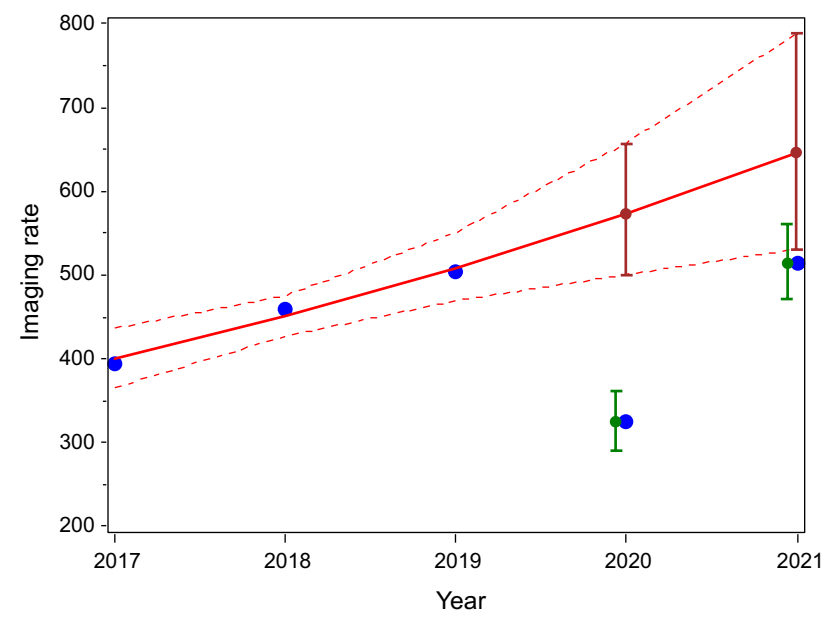

Fig. 1 Observed versus predicted imaging rates. Observed rates for 2017-2021 are plotted in blue. Estimates and predictions for 20172021 based on 2017-2019 data are indicated by the red solid line with red dashed lines for 95\% CIs. Observed rates in 2020 and 2021 (green dots with green lines for 95\% CIs) were lower than predicted (brown dots with brown lines for 95\% CIs)

was also not significantly different from predicted in either year $(2020$ : $\mathrm{OR}=1.89 / 2.28=0.83, p=0.604 ; 2021$ : $\mathrm{OR}=1.73 / 2.83=0.61, p=0.322)($ Fig. $3 \mathrm{~B})$.

\section{Discussion}

This study evaluates two different periods of time during the COVID-19 pandemic. April 2020 was the first full month of the pandemic and coincided with the first wave in the state of Massachusetts with 56,573 statewide cases of COVID-19 diagnosed in April 2020, compared to 7697 cases 1 month earlier. April 2020 also saw the greatest number of deaths from COVID-19 in Massachusetts compared to any other month of the pandemic at 3594 [22]. April 2021 coincided with a third wave of the pandemic, with 49,082 cases of COVID-19 diagnosed in April, and similar numbers 1 month earlier [22]. Although the monthly new caseload of infections was not substantially lower than 1 year earlier, effective vaccines for COVID19 had become available and by April 30, 2020, approximately $35 \%$ of residents in Massachusetts were fully vaccinated and $56 \%$ had received at least one dose [23]. The death toll of the virus had also decreased with April 2021 seeing 480 deaths from COVD-19 statewide [22]. Because of improving public health metrics, by March 22, 2021, Massachusetts entered the final phase of the statewide reopening plan to gradually allow businesses, services, and activities to return to normal [24]. a

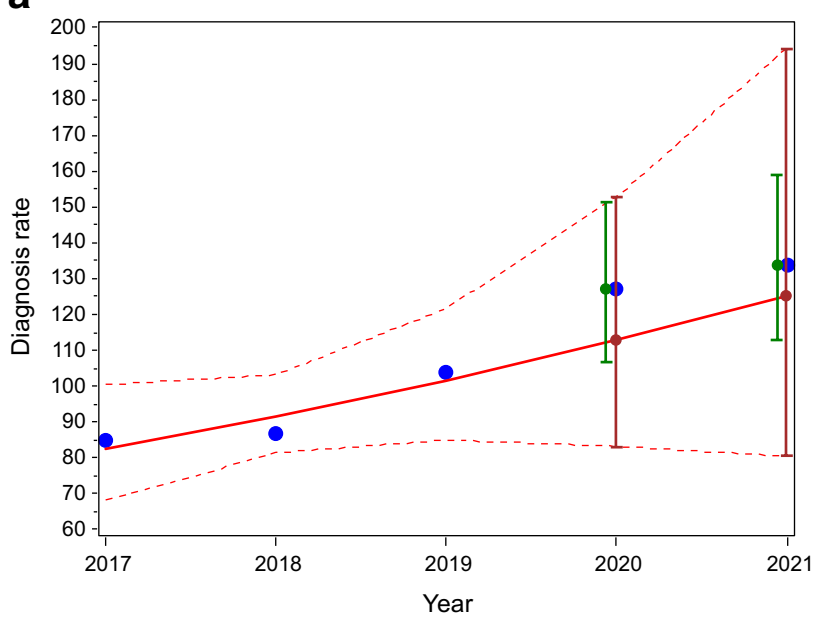

b

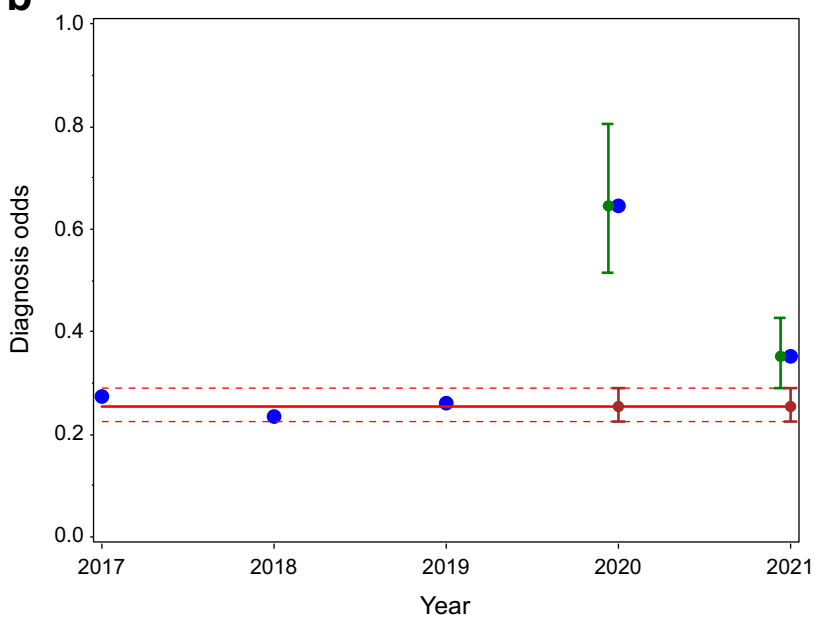

Fig. 2 A Observed versus predicted positive diagnosis rate. The observed rates for all years are plotted in blue. The red solid and dashed lines indicate estimated predictions based on 2017-2019 data with $95 \%$ confidence interval for the 2020 and 2021 rates. The observed rates in 2020 and 2021 (green dot, with green lines indicating confidence interval) were within predicted rates (brown dot and lines). B Observed versus predicted positive diagnosis odds. The observed odds for all years are plotted in blue. Estimates and predictions for 2017-2021 based on 2017-2019 data are indicated by the red solid line with red dashed lines for $95 \%$ CIs. The observed positive diagnosis odds in 2020 and 2021 (green dot, with green lines indicating confidence interval) were higher than expected (brown dot and lines for $95 \%$ CIs)

\section{Imaging volumes}

In the years prior to the pandemic, rates of advanced imaging increased at our institution, consistent with published trends across other pediatric emergency departments [25]. During the pandemic, imaging volumes for our pediatric ED decreased significantly, particularly when compared to predicted volumes based on increasing trends. This finding accords with other studies demonstrating decreases in the 
a

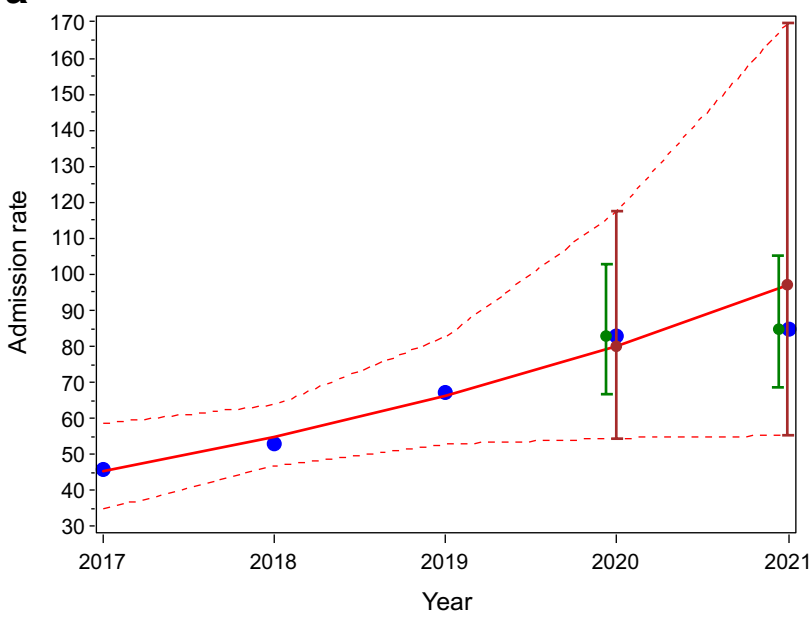

b

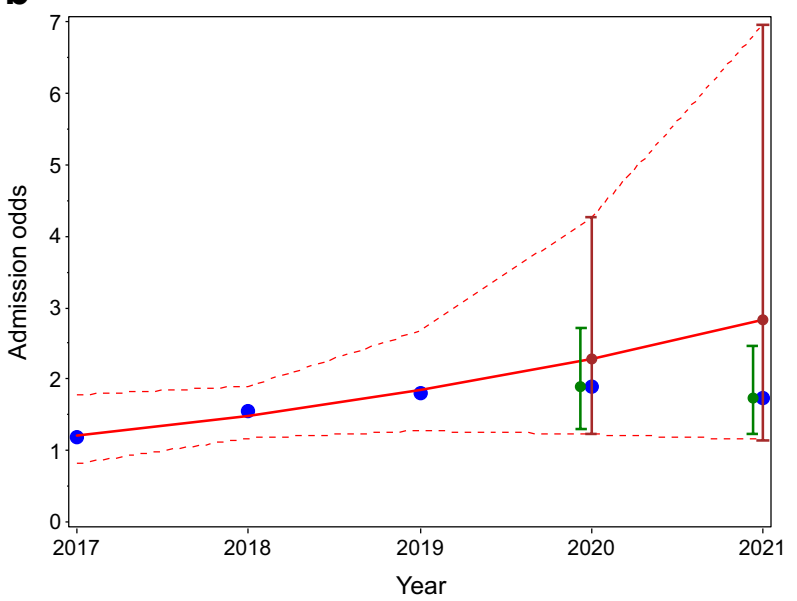

Fig. 3 A Observed versus predicted rate of admission for positive diagnoses. The observed rates for all years are plotted in blue. The red solid and dashed lines indicate estimated predictions based on 2017-2019 data with 95\% confidence interval for the 2020 and 2021 rates. The observed rates in 2020 and 2021 (green dot, with green lines indicating confidence interval) were within predicted rates (brown dot and lines). B Observed versus predicted odds of admission for positive diagnoses. The observed odds for all years are plotted in blue. Estimates and predictions for 2017-2021 based on 20172019 data are indicated by the red solid line with red dashed lines for 95\% CIs. The observed admission odds in 2020 and 2021 (green dot, with green lines indicating confidence interval) were within the expected range of predicted admission odds (brown dot and lines)

overall number of patients presenting to pediatric EDs during the pandemic, both in the US and abroad [14, 17, 26]. Although associated decreases in imaging studies performed in adult EDs have been reported [27-29], to our knowledge, no studies have evaluated similar trends in pediatric emergency radiology nor have incorporated prediction models to compare changes observed during the pandemic.

The decrease in imaging volumes for pediatric ED patients seen in our study correlated with the overall decreased rate at which children presented to the ED during the COVID-19 pandemic. As such, several possible explanations exist for the decreased ED imaging volumes we found. Other reports indicate that fear of exposure to COVID-19 was an important potential factor deterring patients from visiting EDs [12]. Decreased ED patient volumes were similarly described during the Severe Acute Respiratory Syndrome (SARS) pandemic in 2003 and the Middle East Respiratory Syndrome (MERS) outbreak in 2015 [30-32]. Additionally, early in the pandemic, governmental and hospital institutional public announcements discouraged patients from visiting EDs for non-urgent medical issues [3]. Before the pandemic, up to $37-60 \%$ of pediatric patients arriving to EDs in the US reportedly did so for non-urgent reasons [33]. Both US and international reports indicate that a large decrease in lower acuity visits may account for the majority of decreased ED patient volumes during the pandemic $[17,26,34]$. This suggests that many patients appropriately self-triaged and avoided inappropriate use of the ED. For children, physical distancing and school closures may have decreased incidences of non-COVID-related communicable illnesses, with lower incidences of gastroenteritis, otitis, and other viral diseases compared to previous years $[17,34]$. In addition, improved access to telehealth services may have allowed families to avoid some urgent care visits for lower acuity illnesses [14, 35, 36].

The decreased diagnostic imaging volumes in the ED might also have been affected by changes in practice parameters by ED clinicians. One study from an academic pediatric ED reported an association of decreased volumes during the early pandemic with overall shorter time-related patient flow parameters, and fewer patients who left the ED before being seen [14]. This would argue against limited medical resources as a cause for the decrease we observed. One Italian study found a $19 \%$ decrease in negative radiology studies ordered for trauma in a pediatric ED during the first wave of the pandemic, compared to the pre-COVID era [34]. The authors attributed this to possibly more appropriate imaging utilization during the pandemic. However, selection could also have been at the patient level, with those presenting to the ED potentially more likely to have positive radiologic findings compared to before the pandemic. In our ED, there are typically low rates of patients who leave without being seen, and staff resources and access to imaging were not limited during the pandemic. Furthermore, there are robust clinical pathways for ED imaging criteria, which were not affected during the pandemic. Thus, it would seem reasonable to suggest that the threshold for ordering diagnostic imaging studies would not have changed significantly for those patients who did present to our ED during the pandemic.

While the imaging rate in April 2021 remained lower than predicted, the decrease was not as great as that seen earlier in the pandemic. This suggests that, while it was not yet over, 
the decreasing effects of the pandemic on the health and function of the community at that time were accompanied by a shift towards baseline patient behaviors.

\section{Rate of positive imaging and admittance}

Several studies demonstrate that patients who presented to pediatric EDs during the pandemic often presented with higher acuity and rates of complications, suggesting that patients were delaying care [17, 26, 37]. Rates of appendiceal perforation have reportedly been higher in both pediatric and adult studies during the pandemic [38-41]. Unexpectedly decreased diagnoses of acute coronary syndromes and strokes in EDs, associated with increased out-of-hospital mortality rates for these conditions, further suggest that many acute illnesses went undiagnosed [6]. These worrisome trends were reported internationally within nations heavily affected by COVID- 19 .

We found a comparatively increased odds of positive imaging diagnoses during the pandemic, consistent with studies demonstrating increased percentages of higher acuity ED patients during the COVID-19 pandemic. However, given that both the number of observed positive imaging diagnoses and the observed admission rates for those positive studies in April 2020 and April 2021 were within the predicted range based on previous years, it seems probable that children were appropriately presenting to the ED for urgent conditions, and that there were not undue rates of delayed diagnoses or conditions that went undiagnosed. The overall distribution of common indications for imaging was similar across all years.

During the worst months of the pandemic, many pediatric patients were transferred or diverted to dedicated pediatric hospitals such as ours, as general hospitals prepared for excess numbers of adult COVID-19 patients [42, 43]. It is possible that higher acuity patients were consequently transferred or diverted to our ED, potentially increasing our rate of observed positive imaging studies. One academic pediatric ED study reported a higher proportion of referrals and ambulance arrivals during the same period [14]. A Canadian study found that parents were more likely to visit the ED at a dedicated children's hospital rather than at a general hospital, possibly to minimize exposure to other adults with COVID-19 [17].

As with imaging rate, the odds of positive diagnosis in April 2021 were closer to the pre-pandemic value than in April 2020. At the hospital level, this may have been related to changing policies at healthcare facilities, which resumed care for non-urgent visits, and had more infrastructure in place to continue efficient urgent and emergent care. At the patient level, this change may reflect a return to normalcy in patient behavior, namely in the return of lower acuity patients to the ED.

\section{Limitations and further areas of study}

There are several limitations to this study. The 1-month time period studied across years limits the number of radiology studies for analysis. April 2020 was the month of greatest decline in ED patient volume during the pandemic. It therefore likely reflected the time period when patient/family and physician behavior were most affected by the COVID-19 pandemic. Our study focuses on the Emergency Radiology experience during the pandemic and cannot differentiate the effects of patient choice and behavior from potential changes in ED practice patterns, particularly regarding patient imaging. Furthermore, we cannot know with confidence how rates of presentation to the ED were influenced by behavioral changes related to fear and anxiety of exposure to COVID-19, the economic impact of the pandemic resulting in unemployment, and decreased access to healthcare, childcare, and transportation. We may have seen a higher percentage of positive imaging exams during the pandemic compared to prior years because pediatric patients were shifted away from general hospitals that were focusing on adult COVID patients. A broader study of pediatric imaging in the region would be necessary to assess the degree to which such patient transfers or diversions may have influenced our results. Further study is also needed to determine how pediatric ED imaging is affected by evolving developments in the COVID-19 pandemic, as new waves of infections and additional viral variants emerge.

\section{Conclusion}

During the COVID-19 pandemic, significantly fewer ED patients underwent imaging for non-COVID-related illnesses than would have been predicted based on imaging patterns for the immediately preceding 3 years. However, the odds of positive diagnosis increased such that the number of positive diagnoses and rate of hospital admission for those studies were within the expected range based on prior years. This suggests that the pandemic did not result in a substantial number of missed diagnoses or severely delay the diagnosis of non-COVID-related conditions in our pediatric ED, although studies from other institutions have indicated otherwise. Imaging volumes in 2021 suggested a return towards baseline patient behavior as the pandemic conditions improved. Greater understanding of trends in emergency imaging during public health crises is necessary to allow proactive measures to facilitate timely and appropriate radiologic care when crises arise. 
Author contribution The first draft of the manuscript was written by Wendy Kim. Wendy Kim and Delma Jarrett were responsible for project idea, data, collection and analysis. Patrick Johnston performed statistical analysis. Joshua Nagler and Stephen Brown advised and added information regarding clinical perspective and patient access. All authors commented and edited the manuscript.

Availability of data and material The authors declare that they had full access to all of the data in this study and the authors take complete responsibility for the integrity of the data and the accuracy of the data analysis.

Code availability Not applicable.

\section{Declarations}

Ethics approval Ethical approval was waived by the institutional review board.

Consent to participate Written patient or guardian consent was waived.

Consent for publication Not applicable.

Conflict of interest The authors declare that they have no conflict of interest.

\section{References}

1. Moreland A, Herlihy C, Tynan MA et al (2020) Timing of state and territorial COVID-19 stay-at-home orders and changes in population movement - United States, March 1-May 31, 2020. MMWR Morb Mortal Wkly Rep 69:1198-1203

2. CDC (2021) Healthcare workers. https://www.cdc.gov/coronavirus/2019-ncov/hcp/framework-non-COVID-care.html. Accessed 26 Apr 2021

3. CDC (2021) Healthcare facilities: managing operations during the COVID-19 pandemic. https://www.cdc.gov/coronavirus/2019ncov/hcp/guidance-hcf.html. Accessed 26 Apr 2021

4. Hartnett KP, Kite-Powell A, DeVies J et al (2020) Impact of the COVID-19 pandemic on emergency department visits - United States, January 1, 2019-May 30, 2020. MMWR Morb Mortal Wkly Rep 69:699-704. https://doi.org/10.15585/mmwr.mm692 $3 \mathrm{e} 1$

5. Pines JM, Zocchi MS, Black BS et al (2021) Characterizing pediatric emergency department visits during the COVID-19 pandemic. Am J Emerg Med 41:201-204. https://doi.org/10.1016/j. ajem.2020.11.037

6. Santi L, Golinelli D, Tampieri A et al (2021) Non-COVID-19 patients in times of pandemic: emergency department visits, hospitalizations and cause-specific mortality in Northern Italy. PLoS One 16. https://doi.org/10.1371/journal.pone.0248995

7. Lerner EB, Brooke Lerner E, Newgard CD, Clay Mann N (2020) Effect of the coronavirus disease 2019 (COVID-19) pandemic on the U.S. emergency medical services system: a preliminary report. Acad Emerg Med 27:693-699

8. Westgard BC, Morgan MW, Vazquez-Benitez G et al (2020) An analysis of changes in emergency department visits after a state declaration during the time of COVID-19. Ann Emerg Med 76:595-601. https://doi.org/10.1016/j.annemergmed.2020.06.019

9. Zintsmaster MP, Myers DT (2020) Patients avoided important care during the early weeks of the coronavirus pandemic: diverticulitis patients were more likely to present with an abscess on CT. Emerg Radiol. https://doi.org/10.1007/ s10140-020-01854-6

10. Romero J, Valencia S, Guerrero A (2020) Acute appendicitis during coronavirus disease 2019 (COVID-19): changes in clinical presentation and CT findings. J Am Coll Radiol 17:1011-1013. https://doi.org/10.1016/j.jacr.2020.06.002

11. Bugger H, Gollmer J, Pregartner G et al (2020) Complications and mortality of cardiovascular emergency admissions during COVID-19 associated restrictive measures. PLoS ONE 15:e0239801. https://doi.org/10.1371/journal.pone.0239801

12. Lazzerini M, Barbi E, Apicella A et al (2020) Delayed access or provision of care in Italy resulting from fear of COVID-19. The Lancet Child \& Adolescent Health 4:e10-e11

13. Sheath C, Abdelrahman M, MacCormick A, Chan D (2021) Paediatric appendicitis during the COVID-19 pandemic. J Paediatr Child Health. https://doi.org/10.1111/jpc.15359

14. Even L, Lipshaw MJ, Wilson PM et al (2021) Pediatric emergency department volumes and throughput during the COVID-19 pandemic. Am J Emerg Med 46:739. https://doi.org/10.1016/j.ajem. 2020.09.074

15. Beyer CA, Hopper LD, French CE et al (2020) The effect of California's stay-at-home order on trauma patient volume during the Coronavirus disease 2019 pandemic. Annals of Surgery Open 1:e005. https://doi.org/10.1097/AS9.0000000000000005

16. Leichtle SW, Rodas EB, Procter L et al (2020) The influence of a statewide "stay-at-home" order on trauma volume and patterns at a level 1 trauma center in the united states. Injury 51:2437-2441

17. Goldman RD, Grafstein E, Barclay N et al (2020) Paediatric patients seen in 18 emergency departments during the COVID19 pandemic. Emerg Med J 37:773-777. https://doi.org/10.1136/ emermed-2020-210273

18. Bickell NA, Aufses AH Jr, Rojas M, Bodian C (2006) How time affects the risk of rupture in appendicitis. J Am Coll Surg 202:401-406

19. Agresti A (ed) (2013) Categorical data analysis, 3rd edn. John Wiley and Sons Inc, Hoboken, New Jersey

20. SAS Institute Inc. SAS/STAT® 14.1 User Guide. SAS Institute Inc; 2015

21. Burnham KP, Anderson DR (2002) Model selection and multimodel inference: a practical information-theoretic approach, 2nd Edition. Springer Science \& Business Media. Springer, New York. https://doi.org/10.1007/b97636

22. Department of Public Health Archive of COVID-19 cases in Massachusetts

23. Department of Public Health Massachusetts COVID-19 vaccination data and updates

24. Reopening Massachusetts. https://www.mass.gov/info-details/ reopening-massachusetts. Accessed 4 Aug 2021

25. Marin JR, Rodean J, Hall M et al (2020) Trends in use of advanced imaging in pediatric emergency departments, 2009-2018. JAMA Pediatr 174:e202209

26. Chaiyachati BH, Agawu A, Zorc JJ, Balamuth F (2020) Trends in pediatric emergency department utilization after institution of coronavirus disease-19 mandatory social distancing. J Pediatr. https://doi.org/10.1016/j.jpeds.2020.07.048

27. Houshyar R, Tran-Harding K, Glavis-Bloom J et al (2020) Effect of shelter-in-place on emergency department radiology volumes during the COVID-19 pandemic. Emerg Radiol 27:781-784. https://doi.org/10.1007/s10140-020-01797-y

28. Boeken T, Le Berre A, Mebazaa A et al (2020) Non-COVID-19 emergencies: where have all the patients gone? Eur Radiol 30:5220-5221. https://doi.org/10.1007/s00330-020-06961-z

29. Cavallo JJ, Forman HP (2020) The economic impact of the COVID-19 pandemic on radiology practices. Radiology 296:E141-E144. https://doi.org/10.1148/radiol.2020201495 
30. Paek SH, Kim DK, Lee JH, Kwak YH (2017) The impact of middle east respiratory syndrome outbreak on trends in emergency department utilization patterns. J Korean Med Sci 32:1576-1580. https://doi.org/10.3346/jkms.2017.32.10.1576

31. Huang H-H, Yen DH-T, Kao W-F et al (2006) Declining emergency department visits and costs during the severe acute respiratory syndrome (SARS) outbreak. J Formos Med Assoc 105:31-37. https://doi.org/10.1016/S0929-6646(09)60106-6

32. Boutis K, Stephens D, Lam K et al (2004) The impact of SARS on a tertiary care pediatric emergency department. CMAJ 171:13531358. https://doi.org/10.1503/cmaj.1031257

33. Brousseau DC, Hoffmann RG, Nattinger AB et al (2007) Quality of primary care and subsequent pediatric emergency department utilization. Pediatrics 119:1131-1138. https://doi.org/10.1542/ peds.2006-3518

34. Liguoro I, Pilotto C, Vergine M et al (2021) The impact of COVID-19 on a tertiary care pediatric emergency department. Eur J Pediatr. https://doi.org/10.1007/s00431-020-03909-9

35. Patel PD, Cobb J, Wright D et al (2020) Rapid development of telehealth capabilities within pediatric patient portal infrastructure for COVID-19 care: barriers, solutions, results. J Am Med Inform Assoc 27:1116-1120. https://doi.org/10.1093/jamia/ocaa065

36. Lurie N, Carr BG (2018) The role of telehealth in the medical response to disasters. JAMA Intern Med 178:745-746. https:// doi.org/10.1001/jamainternmed.2018.1314

37. Lynn RM, Avis JL, Lenton S et al (2021) Delayed access to care and late presentations in children during the COVID-19 pandemic: a snapshot survey of 4075 paediatricians in the UK and Ireland. Arch Dis Child 106:e8. https://doi.org/10.1136/archdischi ld-2020-319848
38. Place R, Lee J, Howell J (2020) Rate of pediatric appendiceal perforation at a children's hospital during the COVID-19 pandemic compared with the previous year. JAMA Network Open 3:e2027948

39. Snapiri O, Rosenberg Danziger C, Krause I et al (2020) Delayed diagnosis of paediatric appendicitis during the COVID-19 pandemic. Acta Paediatr 109:1672-1676. https://doi.org/10.1111/apa. 15376

40. Orthopoulos G, Santone E, Izzo F et al (2020) Increasing incidence of complicated appendicitis during COVID-19 pandemic. Am J Surg. https://doi.org/10.1016/j.amjsurg.2020.09.026

41. Lee-Archer P, Blackall S, Campbell $\mathrm{H}$ et al (2020) Increased incidence of complicated appendicitis during the COVID -19 pandemic. J Paediatr Child Health 56:1313-1314

42. Fraymovich S, Levine DA, Platt SL (2020) A blueprint for pediatric emergency resource reallocation during the COVID-19 pandemic: an NYC Hospital experience. Pediatr Emerg Care 36:452-454. https://doi.org/10.1097/PEC.0000000000002203

43. DeFazio JR, Kahan A, Fallon EM et al (2020) Development of pediatric surgical decision-making guidelines for COVID-19 in a New York City children's hospital. J Pediatr Surg 55:1427-1430

Publisher's Note Springer Nature remains neutral with regard to jurisdictional claims in published maps and institutional affiliations. 